\title{
Essential role of RSK2 in c-Fos-dependent osteosarcoma development
}

\author{
Jean-Pierre David, ${ }^{1,3}$ Denis Mehic, ${ }^{1}$ Latifa Bakiri, ${ }^{1}$ Arndt F. Schilling, ${ }^{2}$ Vice Mandic, ${ }^{3}$ \\ Matthias Priemel,2 Maria Helena Idarraga,1 Markus O. Reschke,1 Oskar Hoffmann, ${ }^{4}$ \\ Michael Amling, ${ }^{2}$ and Erwin F. Wagner ${ }^{1}$ \\ ${ }^{1}$ Research Institute of Molecular Pathology, Vienna, Austria. ${ }^{2}$ Department of Trauma Surgery, School of Medicine, Hamburg University, \\ Hamburg, Germany. ${ }^{3}$ Deutsches Rheuma-Forschungszentrum, Berlin, Germany. ${ }^{4}$ Institut für Pharmakologie und Toxikologie, \\ Pharmazie-Zentrum, University of Vienna, Vienna, Austria.
}

\begin{abstract}
Inactivation of the growth factor-regulated S6 kinase RSK2 causes Coffin-Lowry syndrome in humans, an $X$-linked mental retardation condition associated with progressive skeletal abnormalities. Here we show that mice lacking RSK2 develop a progressive skeletal disease, osteopenia due to impaired osteoblast function and normal osteoclast differentiation. The phenotype is associated with decreased expression of Phex, an endopeptidase regulating bone mineralization. This defect is probably not mediated by RSK2-dependent phosphorylation of c-Fos on serine 362 in the C-terminus. However, in the absence of RSK2, c-Fos-dependent osteosarcoma formation is impaired. The lack of c-Fos phosphorylation leads to reduced c-Fos protein levels, which are thought to be responsible for decreased proliferation and increased apoptosis of transformed osteoblasts. Therefore, RSK2-dependent stabilization of c-Fos is essential for osteosarcoma formation in mice and may also be important for human osteosarcomas.
\end{abstract}

\section{Introduction}

Bone integrity depends on the proper balance between the activity of the bone-forming cells, osteoblasts, and the bone-resorbing cells, osteoclasts (1). c-Fos, a member of the heterodimeric transcription factor complex AP-1, has been shown to play key roles in modulating both osteoclast differentiation and osteoblast transformation $(2,3)$. Mice lacking c-Fos are osteopetrotic because of a complete block of osteoclast differentiation $(4,5)$. In contrast, mice overexpressing c-Fos develop osteosarcomas, a tumor affecting the osteoblastic lineage (6). Although not essential for osteoblast differentiation, c-Fos is expressed in osteoblasts, and osteogenic factors such as parathyroid hormone (PTH) or parathyroid hormone-related protein (PTHrP) transcriptionally induce $c$-fos $\operatorname{mRNA}(7,8)$.

In addition to the transcriptional control, c-Fos is subject to extensive posttranslational modifications. Phosphorylation of c-Fos on threonine 232 by a not fully characterized kinase called Fos-regulating kinase (FRK) can stimulate its transcriptional activity (9). The role for ERK activation in the posttranscriptional regulation of c-Fos is better characterized. In vitro, both ERK and the ERK/PI3K-dependent kinase RSK2 can phosphorylate the C-terminal domain of c-Fos on serines 374 and 362, respectively. These 2 phosphorylation events stabilize the protein, allowing subsequent phosphorylation by ERK on threonine 325 and threonine 331, thereby modulating c-Fos-induced cell transformation (10-12). Recently, IFN- $\beta$ has been shown to inhibit osteoclast differentiation by posttranscriptionally repressing c-Fos expression in osteoclast progenitors (13). Thus, c-Fos activity can be both tran-

Nonstandard abbreviations used: CLS, Coffin-Lowry syndrome; $\mu \mathrm{CT}$, microcomputed tomography; FRK, Fos-regulating kinase; M-BMM, M-CSF-dependent bone marrow monocyte; PTH, parathyroid hormone.

Conflict of interest: The authors have declared that no conflict of interest exists.

Citation for this article: J. Clin. Invest. 115:664-672 (2005)

doi:10.1172/JCI200522877. scriptionally and posttranscriptionally regulated in osteoblasts and in osteoclasts.

The putative c-Fos kinase RSK2 belongs to the group of broadly expressed protein kinases called ribosomal S6 kinases. RSKs are composed of 2 functional kinase domains connected by a linker, which are sequentially activated by ERK and by the PI3K-dependent kinase PDK1 (14). In addition to directly phosphorylating c-Fos, RSK2-mediated CREB phosphorylation has been shown to be required for $c$-fos transcriptional regulation in response to growth factor stimulation $(15,16)$. Furthermore, RSK2 appears to be involved in chromatin remodeling associated with the activation of the $c$-fos promoter by EGF (17). Importantly, Coffin-Lowry syndrome (CLS), a human X-linked mental retardation disorder associated with progressive skeletal abnormalities, is due to inactivating mutations of RSK2 (18). These observations prompted us to study the function of RSK2 in bone development and to investigate the functional relevance of the posttranscriptional modifications of c-Fos by RSK2 to osteoclast differentiation and to osteosarcoma formation.

\section{Results}

c-Fos phosphorylation by RSK2 appears to be dispensable in osteoclasts. Since serine 362 in the C-terminal domain of c-Fos has been proposed to be an RSK2 phosphorylation site essential for c-Fos transactivation activity, we first generated an antibody directed against c-Fos phospho-serine 362 (Figure 1A). This antibody recognized a band corresponding to the molecular weight of c-Fos in $3 \mathrm{~T} 3$ fibroblasts. The reactive band was also detected when the adjacent serine 374 was mutated to alanine, but it was absent when serine 362 was mutated to alanine (Figure 1A). Reblotting with a specific anti-c-Fos antibody confirmed that the protein recognized by the anti-phospho-serine 362 was indeed c-Fos (Figure 1A). Immunostaining of $\mathrm{M}$-CSF-dependent bone marrow monocytes (M-BMMs) further showed that c-Fos was phosphorylated on serine 362 in response to M-CSF (Figure 1B). Moreover, using immunoprecipitation 

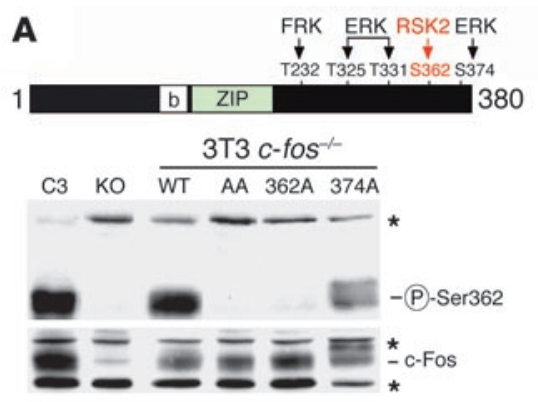

B
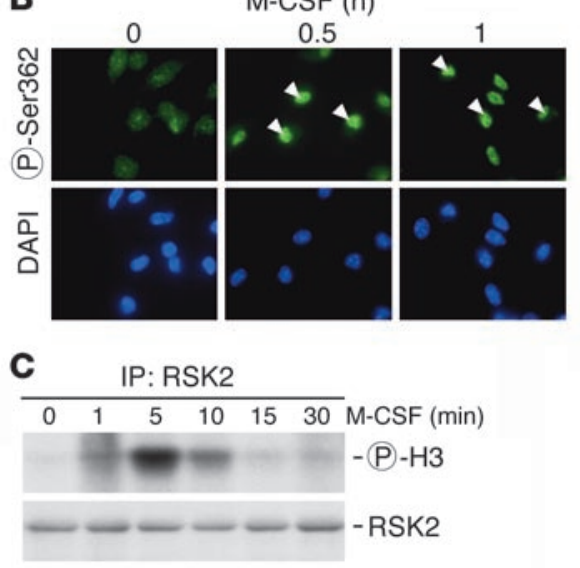
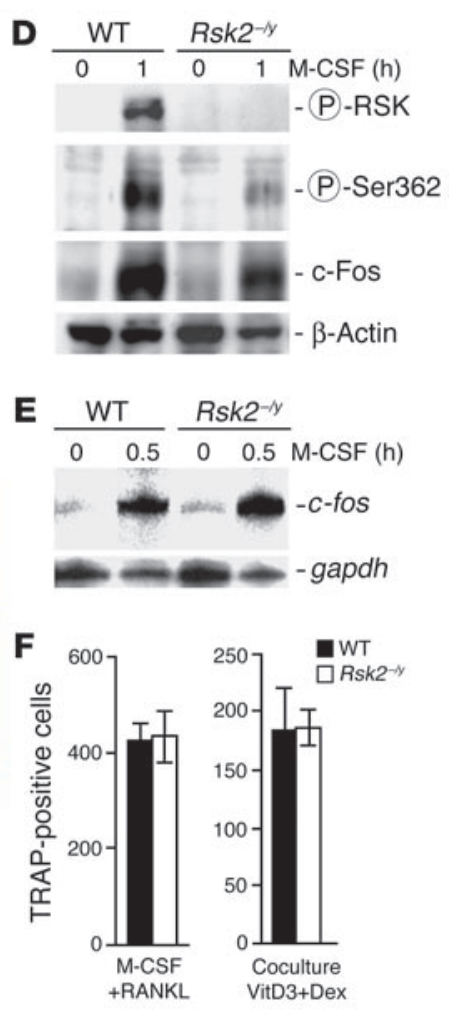

\section{Figure 1}

c-Fos phosphorylation by RSK2 is not required for osteoclast differentiation. (A) Characterization of the specific antibody directed against c-Fos phospho-serine 362. The position of the putative RSK2 phosphorylation site in the C-terminal domain of c-Fos is indicated in red. Other proposed phosphorylation sites, kinases that phosphorylate them, and the relative positions of the DNA binding and dimerization domain (bZIP) are also indicated. The following were analyzed by Western blot: c-Fos expression and phosphorylation on serine 362 in c-fos-deficient 3 T3 fibroblasts re-expressing wild-type c-Fos (WT) or mutated forms in which serine 362 has been replaced by alanine $(362 \mathrm{~A})$ and/or serine 374 replaced by alanine (AA and $374 \mathrm{~A})$. A c-Fos osteosarcoma cell line (C3) and the parental 3T3 (KO) were used as controls. *Nonspecific reacting bands. (B) C-Fos phosphorylation in M-CSF-stimulated M-BMMs. Immunostaining using the phospho-serine $362 \mathrm{c}$-Fos antibody. Arrowheads indicate immunopositive nuclei, visualized using DAPI. Magnification, $\times 20$. (C) RSK2 activation in M-CSF-stimulated M-BMMs. Immunoprecipitation kinase assay using an antibody directed against RSK2 and histone $\mathrm{H} 3$ as a substrate. (D) C-Fos expression and phosphorylation on serine 362 in $R s k 2^{-l y}$ M-BMMs compared with wild-type. RSK2 activation was analyzed using anti-phospho-RSK2; $\beta$-actin was used as loading control. (E) Expression of $c$-fos mRNA in M-BMMs lacking RSK2, analyzed by RNase protection assay; gapdh was used as loading control. (F) Left: Differentiation, induced by M-CSF and RANKL, of Rsk2-ly or wild-type monocytes into multinucleated osteoclasts (TRAP-positive cells). Right: Osteoclastogenic properties of calvarial osteoblasts isolated from $R s 2^{-l y}$ or wild-type newborn littermates were analyzed by coculture with wild-type bone marrow cells stimulated by 1,25-dihydroxyvitamin $D_{3}$ (VitD3) and dexamethasone (Dex).

kinase assays, we demonstrated that RSK2 was expressed and became activated in M-BMMs (Figure 1C). These data suggest that M-CSF-induced RSK2 activation could be involved in the posttranscriptional regulation of c-Fos in osteoclast progenitors. We therefore determined whether RSK2 is involved in the posttranscriptional regulation of c-Fos in osteoclasts. Mice lacking the X-linked gene Rsk2 are viable and fertile $(19,20)$. A strong decrease in M-CSF-induced c-Fos expression was observed in Rsk2deficient M-BMMs (Figure 1D). Interestingly, in the absence of
RSK2, residual c-Fos phosphorylation on serine 362 was detected (Figure 1D). RNase protection assays demonstrated that $c$-fos transcriptional regulation by M-CSF was not altered in M-BMMs lacking RSK2 (Figure 1E). These data indicate that RSK2 contributes to but is not essential for the transcriptional and posttranscriptional regulation of c-Fos expression in osteoclast progenitors. As a consequence, Rsk2 deficiency had no effect on osteoclast differentiation or on the resorbing activity, when osteoclast differentiation was induced by M-CSF and RANKL in vitro (Figure $1 \mathrm{~F}$ and data not shown).

In vivo osteoclastogenesis is known to be supported by cells of the osteoblastic lineage that synthesize osteoclastogenic molecules such as RANKL. We therefore analyzed the osteoclastogenic properties of Rsk2-deficient osteoblasts. No change in osteoclast differentiation could be observed when wild-type bone marrow cells were cocultured with primary osteoblasts isolated from the calvaria of newborn $R s k 2^{-1 y}$ mice compared with osteoblasts from wildtype littermates (Figure 1F). Furthermore, Rsk2 deficiency did not significantly affect the levels of RANKL and $O P G$ mRNA expression nor their regulation by dexamethasone and 1,25-dihydroxyvitamin $\mathrm{D}_{3}$ in primary osteoblasts (data not shown). Thus, RSK2 is essential neither for osteoclast differentiation nor for the osteoclastogenic function of osteoblasts in vitro.

RSK2 regulates bone mass. Since humans carrying inactivating mutations of RSK2 suffer from multiple bone defects, we studied the role of RSK2 in bone development in vivo by performing histomorphometric analyses of male and female Rsk2-deficient mice. While no change in bone mass was detectable in the absence of Rsk2 in 2- and 4-week-old males, we observed a significant decrease in bone mass in older mice (Figure 2A). A similar phenotype was also present in females, where the bone defect was first observed at 4 weeks of age (data not shown). No differences in the number of osteoclasts were detected, and osteoblast differentiation was also unaffected, as shown by similar numbers of osteoblasts and osteocytes found in 7-month-old Rsk2-ly and wildtype littermates (Figure 2B). The low-bone-mass phenotype was of functional importance, since a significant decrease in the diameter of the long bones and a decrease in biomechanical competence of Rsk2-deficient bones were measured (Figure 2C). Dynamic histomorphometry demonstrated a substantial decrease in bone-formation rate in these mice (Figure 2D), whereas no defect in resorption by osteoclasts was detected by measurement of the amount of deoxypyridinoline in the urine (data not shown). Finally, no change in the width and in the organization of the growth plate was observed in Rsk2-deficient mice, which indicates that the bone defect is not secondary to a developmental defect affecting chondrocyte differentiation (Figure 2E). These data suggest that Rsk2-deficient mice are osteopenic because of a functional defect of osteoblasts.

We next analyzed the expression of markers of osteoblast differentiation in adult mice by in situ hybridization. The expression of 
A
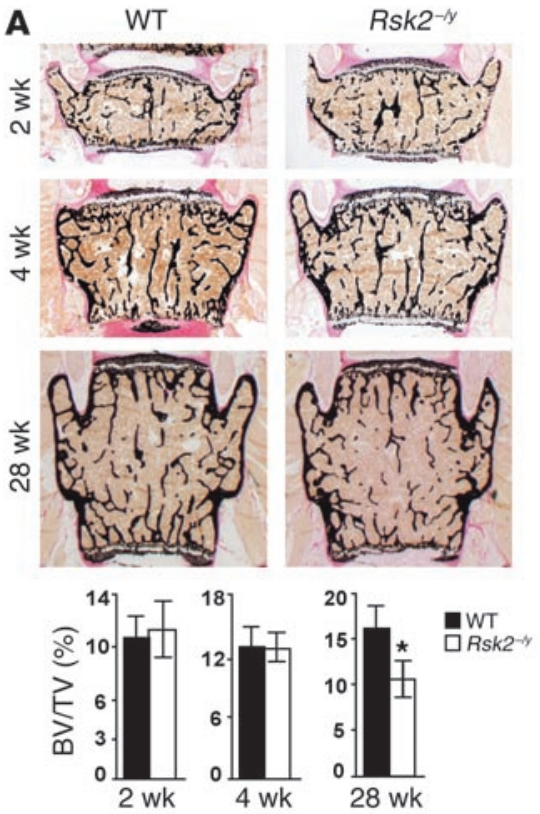

Rsk2 $^{-1 y}$
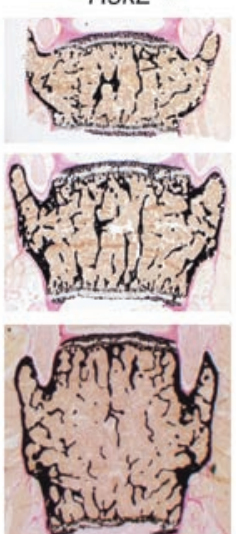

B
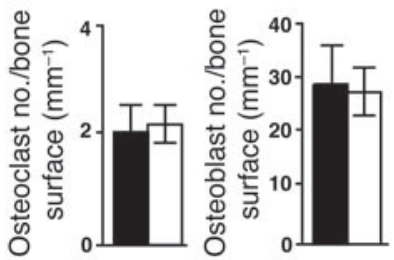

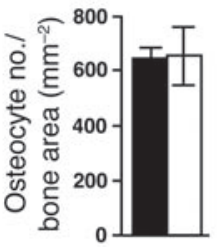

C

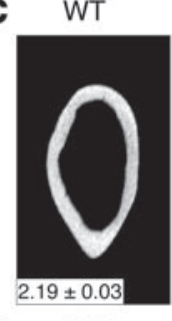

D

WT

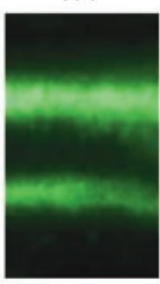

E

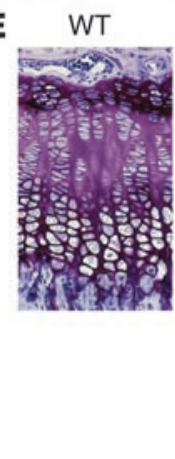

Rsk2-y $^{-1 y}$

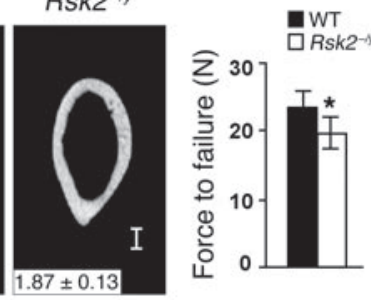

Rsk2 $^{-1 y}$
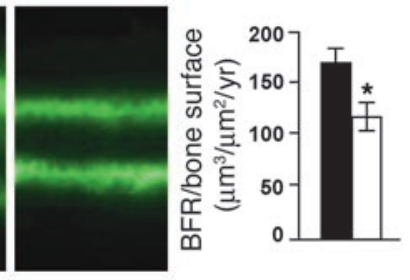

Rsk2-y

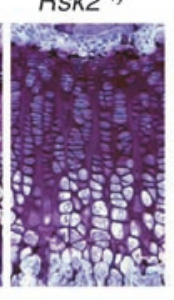

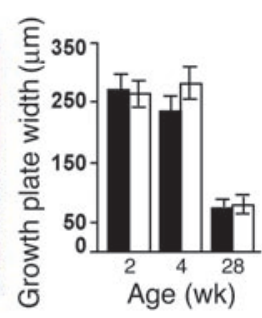

\section{Figure 2}

Rsk2-deficient mice are osteopenic. (A) Histological analysis of vertebral bodies of 2-, 4-, and 28-week-old male wild-type and $R s k 2^{-/ y}$ littermates and quantification of the bone volume (BV) relative to total volume (TV). (B) In vivo quantification of osteoclast, osteoblast, and osteocyte numbers in 28-weekold mice. (C) Decreased femoral thickness (left panel) and decreased biomechanical competence (right panel) of bones lacking Rsk2. Bone diameters in millimeters are indicated in the lower left corners. (D) Determination of the bone-formation rate. Rsk2-deficient and wild-type littermates were injected twice with calcein at 1 -week intervals, and the distance between the 2 lines of fluorochrome (left panel) was measured for assessment of bone-formation rate (BFR) (right panel). (E) Toluidine blue staining of growth plates from 2-weekold wild-type and Rsk2-ly littermates (left panel), and quantitation of growth plate width in 2-, 4-, and 28-week-old mice. ${ }^{*} P<0.05$. Magnification, $\times 25$. type I collagen, osteocalcin, and osteopontin was not affected by the absence of RSK2 in the growth plate, either in the trabecular bone of the primary spongiosa or in the cortex (Figure 3A and data not shown). In contrast, the expression of Phex, an endopeptidase involved in the regulation of mineralization $(21,22)$, was undetectable in the cortex of Rsk2-deficient mice (Figure 3A). To determine whether decreased Phex expression could in part explain the bone defect observed in the absence of RSK2, we compared the mineralization in the cortex of Rsk2-/y mice with that in the cortex of $H y p^{-/ y}$ mice carrying an inactivating mutation of Phex (23). A significant proportion of bone matrix remained unmineralized in the cortex but not in the trabecular bone of Rsk2-deficient mice (Figure 3B). A similar but more severe mineralization defect, which was also more pronounced in the cortex than in the trabecular bone, was observed in $\mathrm{Hyp}^{-1 y}$ mice (Figure 3B and data not shown). However, no significant changes in phosphate contents were detected in the serum and urine of Rsk2-deficient mice (data not shown), which indicates that the mineralization defect is not secondary to a defect in renal phosphate reabsorption but is likely due to decreased Phex expression in cortical bone.

To analyze the cellular mechanisms underlying the osteoblast defect in Rsk2-deficient mice, primary osteoblasts were isolated from wild-type and Rsk2-deficient littermates. Treatment of primary osteoblasts with known bone anabolic molecules such as IGF-1, PTH 1-34, and with BMP2 resulted in a rapid activation of RSK2 and ERK (Figure 3C and data not shown). No difference in cell doublings and Ki67 staining was observed (Figure 3, D and E), and TUNEL staining revealed no changes in apoptosis between wildtype and mutant osteoblasts (Figure 3E). However, in the absence of RSK2, alkaline phosphatase activity was significantly affected, and a drastic reduction in the number of mineralized bone nodules as measured by alizarin red staining was observed (Figure 3F). Moreover, the induction of mineralization by osteoblasts stimulated by IGF-1 was impaired in the absence of RSK2 (Figure 3G). These data indicate that the osteopenia in Rsk2-deficient mice is likely due to a cell-autonomous functional defect of osteoblasts, rather than an effect on osteoblast proliferation or survival.

RSK2 is essential for development of c-Fos-induced osteosarcomas. The osteoblastic phenotype observed in Rsk2-deficient mice and the fact that RSK2 can phosphorylate c-Fos on serine 362 suggested that c-Fos phosphorylation by RSK2 could be involved in the development of c-Fos-induced osteosarcomas. Therefore, we generated $c$-fos transgenic mice lacking RSK2 (Figure 4A). As previously described, H2-c-fosLTR transgenic mice develop osteosarcomas in all bones of the body (6). Interestingly, osteosarcomas were initially also detected in all bones of H2-c-fosLTR/Rsk2- $/ y$ mice (Figure 4, B and $\mathrm{C}$ ). Whereas osteosarcomas increased in size with age in $\mathrm{H} 2$ $c$-fosLTR mice, the tumor area did not significantly increase in mice lacking RSK2 (Figure 4B). Microcomputed tomography and quantitative analyses confirmed that, while the tumor incidence was similar in H2-c-fosLTR and H2-c-fosLTR/Rsk2-/y mice, the tumor burden was drastically reduced in the absence of RSK2 (Figure 4C). Moreover, von Kossa staining revealed that the mineralization inside and outside the tumors was severely impaired, confirming the function of RSK2 in the regulation of bone mineralization (Figure 4D). These data indicated that, while overexpression of c-Fos in osteoblasts does not overcome the RSK2-dependent bone mineralization defect, RSK2 activity is essential for c-Fos- 


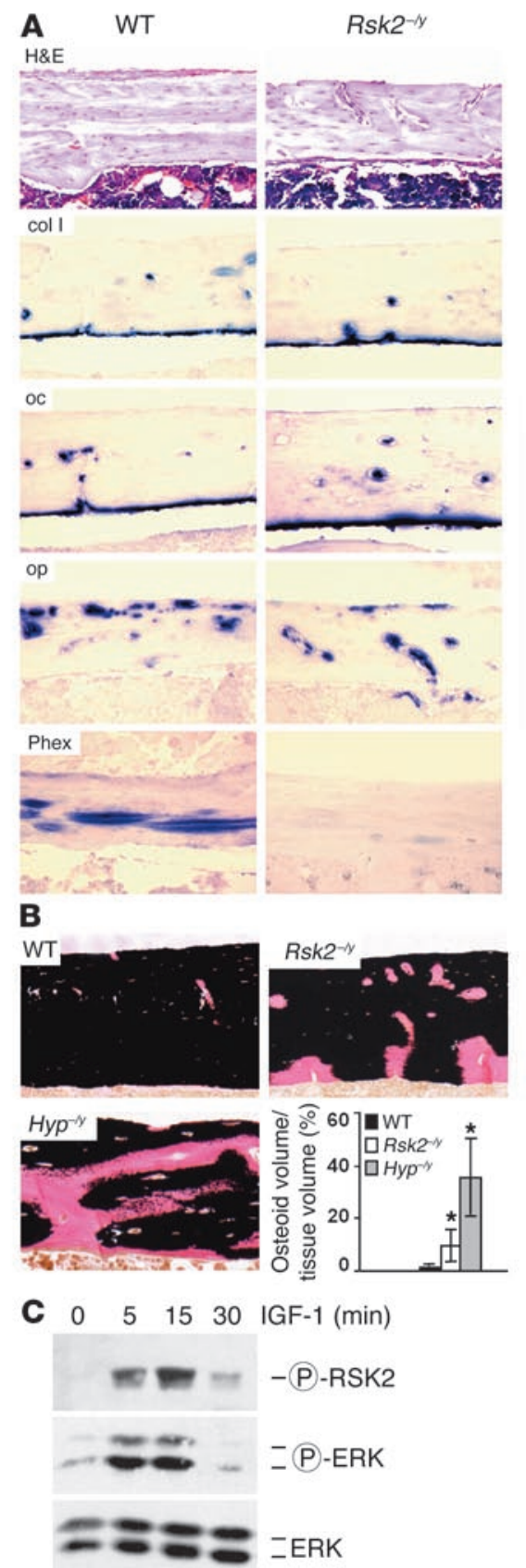

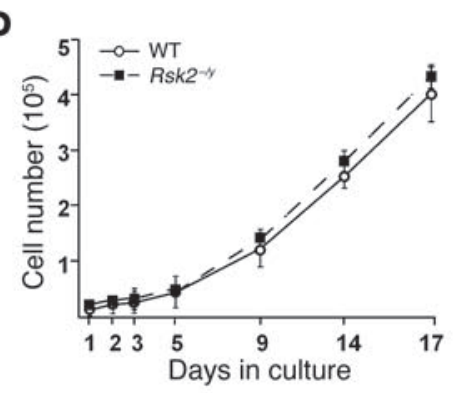

E
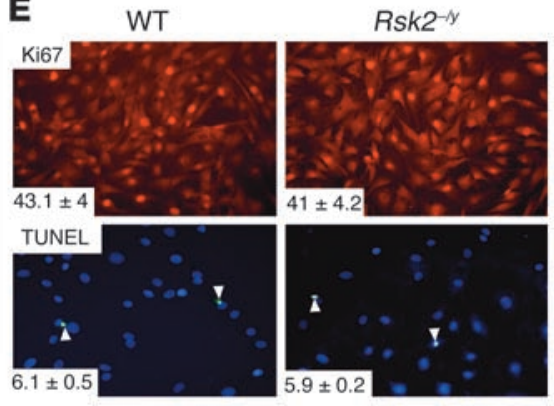

F $\quad W T$

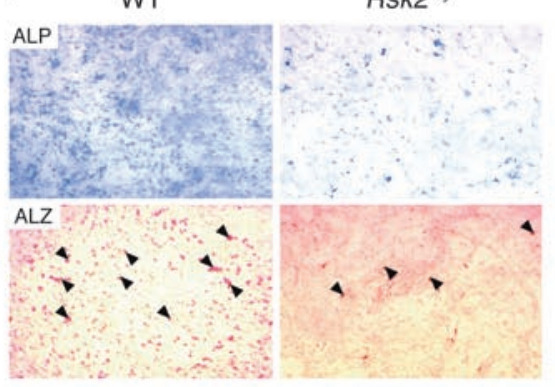

G

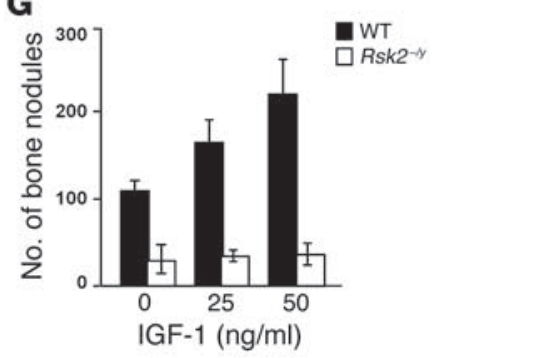

\section{Figure 3}

Molecular and cellular analysis of the bone defects in Rsk2-deficient mice. (A) Analysis of type I collagen (col I), osteocalcin (oc), osteopontin (op), and Phex expression in the cortical bone of 8-week-old wild-type and Rsk2-ly littermates. (B) Analysis of osteoid volume in 28-week-old wildtype and Rsk2-ly littermates compared with $H y p^{-l y}$ mice (24 weeks old). ${ }^{*} P<0.05$. (C) Analysis of RSK2 and ERK activation in primary osteoblast cultures stimulated by IGF-1. RSK2 and ERK activation was analyzed by blotting with phospho-specific antibodies, and the loading was controlled by reblotting with total ERK antibody. (D) Proliferation curve of primary Rsk2-deficient osteoblasts compared with wild-type. (E) Determination of proliferation and apoptotic indices in Rsk2-deficient and wild-type osteoblasts in vitro. Arrowheads indicate TUNEL-positive cells. Quantifications are indicated in the lower left corners. Magnification, $\times 10$. (F) In vitro differentiation of Rsk2-deficient osteoblasts compared with wild-type. Alkaline phosphatase activity (ALP) and bone nodule mineralization by alizarin red staining (ALZ) are shown. Arrowheads indicate alizarin red-positive bone nodules. Magnification, $\times 5$. (G) Effect of IGF-1 on bone nodule mineralization by Rsk2-deficient osteoblasts compared with wild-type.

Thus, the development and the progression of osteosarcomas in c-fos transgenic mice are not due to increased circulating PTH levels.

Progression of osteosarcomas requires RSK2dependent c-Fos posttranscriptional regulation. We next assessed whether decreased tumor formation in the absence of Rsk2 was due to a decrease in c-Fos expression in vivo. RSK2 was found to be expressed in H2-c-fosLTR transgenic tumors but was absent in tumors from H2-c-fosLTR/Rsk2-/y mice (Figure 5A). Importantly, c-Fos was detected in tumors from H2-c-fosLTR transgenic mice and was found phosphorylated on serine 362 (Figure $5 \mathrm{~A})$. In contrast, c-Fos expression was drastically decreased and no phosphorylation on serine 362 was detected in tumors isolated from H2-c-fosLTR/Rsk2-/y mice (Figure 5A). mediated tumor development. It is worth noting that, despite unchanged expression of RANKL and OPG, the small tumors lacking RSK2 had decreased numbers of osteoclasts (Supplemental Figure 1; supplemental material available online with this article; doi:10.1172/JCI200522877DS1), whereas increased osteoclast numbers were proposed to be involved in the development of c-Fosinduced osteosarcomas $(6,24)$. This suggests that active bone resorption is necessary for the progression of osteosarcomas.

It has recently been shown that long-term treatment with PTH might lead to the development of osteosarcomas in rats (25). Therefore, we measured the level of circulating PTH in H2-c-fosLTR, Rsk2-deficient, and wild-type mice. We did not detect any difference in PTH levels in the blood of c-fos transgenic mice or Rsk2deficient mice compared with wild-type mice (data not shown).
To better characterize the cellular mechanisms that could explain the reduction of c-Fos-induced osteosarcomas in the absence of Rsk2, we quantified proliferation and apoptosis within the tumors. Ki67 staining revealed that cell proliferation was decreased in tumors from H2-c-fosLTR/Rsk2-/y mice compared with control littermates (Figure 5B). Furthermore, TUNEL staining demonstrated increased numbers of apoptotic cells in Rsk2-deficient osteosarcomas (Figure 5B).

We next analyzed c-Fos phosphorylation on serine 362 in c-Fosdependent osteosarcomas. Western blot analysis indicated that c-Fos was expressed and phosphorylated on serine 362 in all cell lines isolated and established from c-Fos-dependent osteosarcomas (6), while it was barely detectable in exponentially growing wild-type primary osteoblasts even upon IGF-1 or PTH treatment 
A
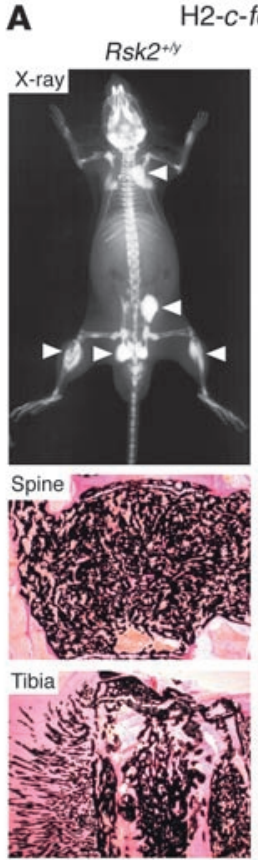

B $\left.{ }^{80}\right\rceil \mathbf{H} 2-c$-fosLTR/Rsk2*y

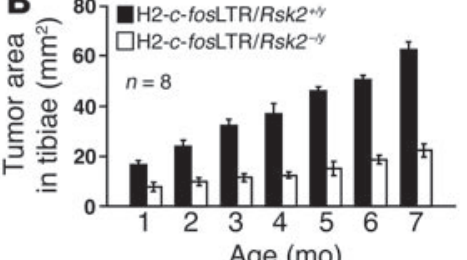

C
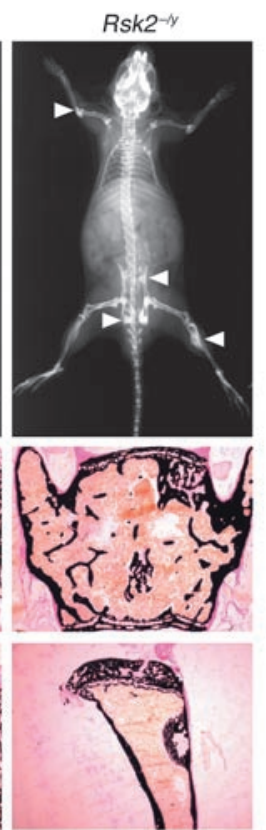

Age (mo)

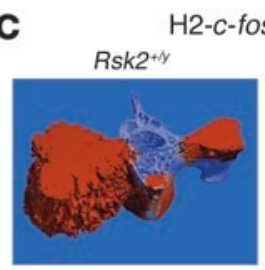

H2-c-fosLTR
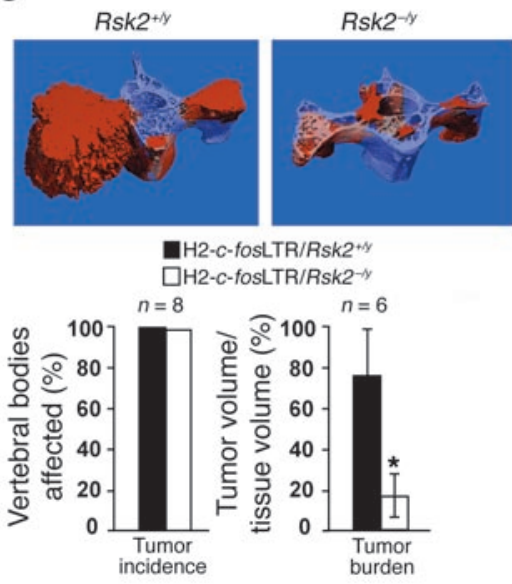

D
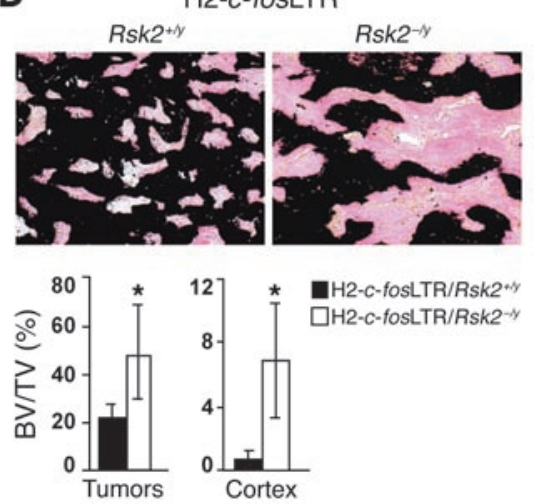

\section{Figure 4}

RSK2 is essential for c-Fos-induced tumor progression. (A) X-ray analysis of 7-month-old $\mathrm{H} 2-$ c-fosLTR and H2-c-fosLTR/Rsk2-/y littermates. Histological analyses of bone sections from vertebral bodies and tibiae. White arrowheads indicate the osteosarcomas. Magnification, $\times 25$. (B) Kinetic analysis of the development of osteosarcomas in $\mathrm{H} 2$-c-fosLTR/Rsk2+/y and H2-c-fosLTR/Rsk2-ly littermates; $n$ indicates the number of tumors measured in tibiae at the indicated age. (C) Top: Microcomputed tomography analysis of vertebrae of 7-month-old H2-c-fosLTR/Rsk2+/y and H2-c-fosLTR/Rsk2-ly littermates. Magnification, $\times 5$. Bottom: Quantification of tumor incidence (percentage of affected vertebrae) and tumor burden (tumor volume relative to tissue volume) in vertebral bodies; $n$ indicates the number of tumor-affected vertebrae measured for tumor burden. (D) Top: Analysis of osteoid volume in tumors of wild-type and $R s k 2^{-1 y}$ littermates. Magnification, $\times 200$. Bottom: Quantification of the osteoid volume in the tumors and in the cortex. ${ }^{*} P<0.05$.
(Figure 5C and data not shown). Interestingly, c-Fos protein levels were drastically reduced and serine 362 phosphorylation totally abolished in primary cells isolated from tumors of H2-c-fosLTR/ $R s k 2^{-/ y}$ mice compared with tumor cells isolated from wild-type littermates (Figure 5C). Northern blot analyses demonstrated that the absence of RSK2 did not affect the expression of $c$-fos mRNA in the long bones (Figure 5C). Thus, RSK2 appears to be essential for c-Fos posttranscriptional stability in c-Fos-induced osteosarcomas. To test this, we analyzed the effect of blocking protein degradation in primary and established cell lines isolated from osteosarcomas of $c$-fos transgenic mice lacking RSK2. Addition of the inhibitor of proteasome activity MG132 was able to stabilize c-Fos expression without inducing phosphorylation (Figure 5D and data not shown). These data demonstrate that RSK2-mediated phosphorylation of c-Fos on serine 362 in the tumors protects c-Fos from proteasome-mediated degradation.

To determine whether the lack of RSK2 activity directly affects survival and proliferation of tumor cells, we analyzed the effect of inhibiting RSK2 activation on apoptosis and proliferation of primary cells isolated from $c$-fos transgenic mice. Upon addition of RO 31-8220, a chemical inhibitor of RSK2 (26), c-Fos protein levels were decreased (Figure 5E). Furthermore, increased apoptosis and decreased proliferation were observed in the primary cells in culture (Figure 5F), which is similar to the in vivo situation (Figure 5B). Thus, impaired development of c-Fos-dependent osteosarcomas in the absence of RSK2 likely results from the combined effect of decreased proliferation and increased apoptosis of c-Fos-transformed osteoblasts.

\section{Discussion}

The growth factor-regulated ribosomal S6 kinase RSK2 appears to be essential for osteoblast function, since mice lacking RSK2 are osteopenic because of a cell-autonomous defect in osteoblast activity. In contrast, osteoclast differentiation was unaffected in vivo and in vitro in the absence of RSK2. However, the development of c-Fos-induced osteosarcomas was found to be dependent on RSK2-mediated c-Fos posttranscriptional regulation. Thus, important functions of c-Fos are determined by RSK2-mediated posttranscriptional mechanisms, which may also be required in human osteosarcomas and in CLS.

Humans carrying mutations of RSK2 suffer from CLS, an $\mathrm{X}$-linked mental retardation condition associated with progressive skeletal deformities and osteopenia, delayed bone age, and delayed fontanelle closure (18). It has been shown that mice lacking RSK2 also have impaired learning and cognitive functions (19). Interestingly, mice lacking c-fos in the CNS have similar learning and behavior deficits (27). Moreover, Rsk2-deficient mice are osteopenic, and it has been shown that some of the skeletal phenotypes observed in CLS are caused by the lack of phosphorylation of ATF 4 by RSK2 (20). We now demonstrate that the osteopenia in Rsk2-deficient mice is also due to a postdevelopmental defect in osteoblast activity. In adult Rsk2-deficient mice, the expression of osteoblast markers of differentiation was not affected, whereas the expression of the mineralization marker Phex was abolished in cortical bone. The Phex mutation in humans is responsible for the development of $\mathrm{X}$-linked hypophosphatemic rickets and of adult-onset vitamin Dresistant hypophosphatemic osteomalacia $(21,22)$. These diseases 
A
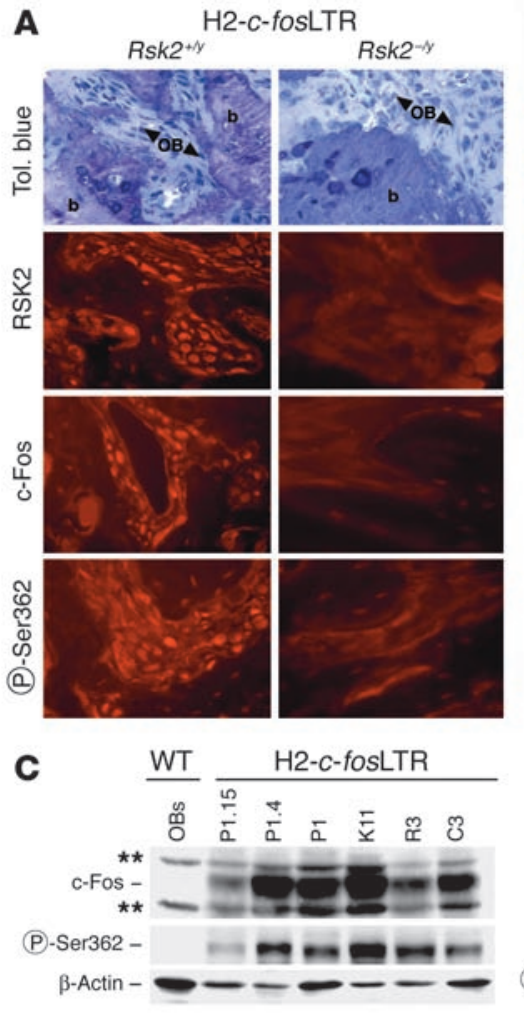

D

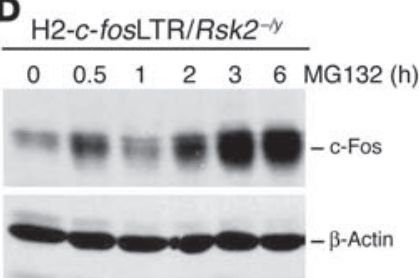

E

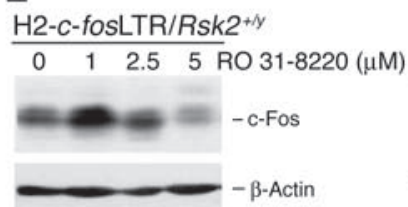

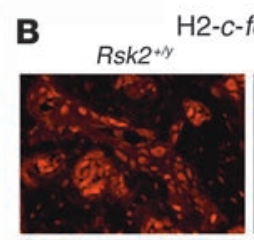
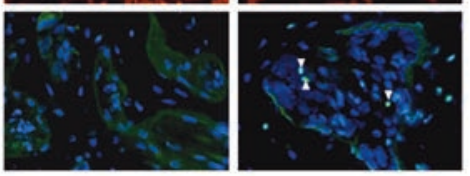

- $\mathrm{H} 2-c$-fosLTR/Rsk2*y
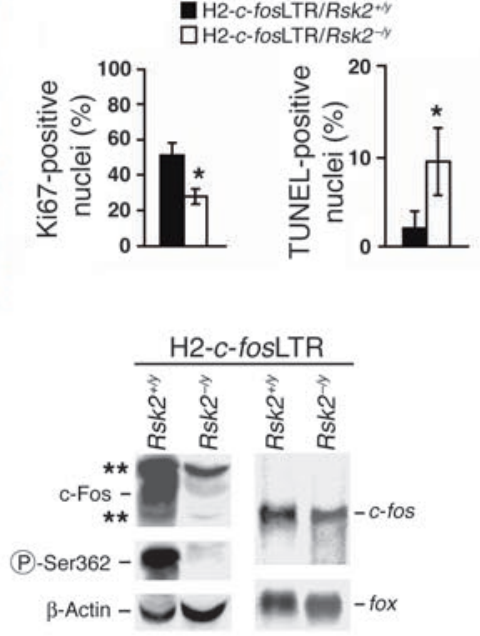

Rsk2-y $^{-1}$

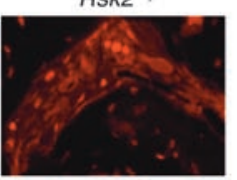

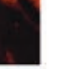

$c$-fosLTR/Rsk2-ly mice. Top row: toluidine blue : toluidine blue staining; second row, RSK2; third row, c-Fos; bottom row, P-Ser362. b, bone; arrows, osteoblasts. (B) Determination of proliferation and apoptotic indices in tumor section of H2-c-fosLTR/Rsk2+/y and H2-c-fosLTR/Rsk2-ly littermates. Proliferation was determined by counting of the proportion of Ki67-positive cells within the tumor, and the apoptotic indices were determined by counting of the proportion of TUNEL-positive cells (arrowheads) within the tumor. ${ }^{*} P<0.05$. (C) Left: Western blot analysis of c-Fos expression and phosphorylation on serine 362 in primary osteoblasts (OBs) and in cell lines (P1.15, P1.4, $\mathrm{P} 1, \mathrm{~K} 11, \mathrm{R} 3$, and C3) established from C-Fos-induced osteosarcomas of various origins ( $P$, pelvis; $K$, long bone; R, ribs; C, calvaria). Right: Western blot analysis of c-Fos expression and phosphorylation in primary cells isolated from tumors of $\mathrm{H} 2-c-f o s L T R / R s k 2^{+/ y}$ and H2c-fosLTR/Rsk2-ly littermates and Northern blot analysis of c-Fos expression in long bones of H2-c-fosLTR/Rsk2+/y and $\mathrm{H} 2-c$-fosLTR/Rsk2-ly littermates. Loading was controlled by analysis of $\beta$-actin and fox expression, respectively. Double asterisks indicate nonspecific bands. (D) Effect of inhibition of proteasome activity by MG132 on c-Fos expression in primary cells isolated from $\mathrm{H} 2-\mathrm{c}$-fosLTR/Rsk2-ly tumors (left) and in a cell line established from H2-c-fosLTR/Rsk2-ly tumors (right). (E) Effect of RSK2 inhibitor (RO 31-8220) on c-Fos expression in primary cells isolated from tumors of H2-c-fosLTR/Rsk2+ly mice. (F) Effect of RSK2 inhibitor on apoptosis and proliferation of primary cells isolated from tumors of $\mathrm{H2}$ c-fosLTR/Rsk2+/y mice.

Bone mineralization of Rsk2-deficient osteoblasts was affected in vivo and in vitro. Phex is mainly expressed in osteocytes and in selected osteoblasts (29), and its expression in bone is known to decrease with age, reaching a very low level in the adult (30, 31). It is conceivable that decreased Phex expression might be responsible for decreased mineralization observed in the cortical bones of Rsk2-deficient mice. Whether RSK2-dependent Phex expression is also involved in the reduced bone mass and reduced bone are characterized by renal phosphate wasting and cell-autonomous defects in osteoblast-mediated mineralization (28). A similar patchy mineralization was observed in the cortex of Rsk2-deficient and of Phex-deficient (Hyp) mice, although the mineralization defect was less severe in Rsk2-deficient mice. In both mutant mice a stronger phenotype was observed in the cortex than in trabecular bone (29). Interestingly, Phex overexpression in osteoblasts fails to rescue the hypophosphatemia and to fully rescue the mineralization defect observed in Hyp mice (28). These data indicate that the bone phenotype observed in Hyp mice is due not solely to an intrinsic defect in mineralization by osteoblasts, but also to the systemic hypophosphatemia. The difference between the severities of the mineralization defects observed in Hyp mice and in mice lacking Rsk2 may be explained by the fact that no signs of phosphate wasting were observed in the absence of RSK2. formation in the trabecular bone remains to be established. It has recently been suggested that the reduced bone mass in Rsk2-deficient trabecular bone is caused by decreased type I collagen synthesis (20). These data were supported by in vitro studies showing a posttranscriptional decrease in type I collagen synthesis, when Rsk2- or ATF4-deficient osteoblasts were cultured in media lacking nonessential amino acids (20). While a possible role for altered Phex expression in CLS patients or impaired RSK2 activation in the development of X-linked hypophosphatemia should be explored in future experiments, these data provide substantial evidence that the progressive bone defect associated with CLS is most likely due to a cell-autonomous defect in the function and not in the differentiation of osteoblasts. The development of osteosarcomas in c-fos transgenic mice and the expression of c-Fos in human osteosarcomas $(32,33)$ suggest that $\mathrm{c}$-Fos could be the substrate 
mediating RSK2 function in osteoblasts. However, c-Fos is likely not the main substrate mediating RSK2 function in osteoblasts, since $c$-fos-deficient osteoblasts appear to be functional (34). Moreover, c-Fos protein was barely detectable in exponentially growing and in IGF-1- or PTH-stimulated primary osteoblasts, while osteogenic molecules can induce $c$-fos transcription in osteoblasts $(7,8)$. Importantly, proliferation and cell death observed in c-Fos-induced osteosarcomas were dependent on RSK2, although the absence of RSK2 did not affect these parameters in nontransformed osteoblasts. In addition, overexpression of c-Fos did not rescue the RSK2-dependent osteopenia outside the tumors.

Whereas c-Fos phosphorylation by RSK2 may not be important for normal osteoblast function, RSK2 is essential for osteosarcoma development to maintain high levels of c-Fos protein. The c-Fos protein is known to be unstable and can be rapidly degraded by a proteasome-dependent mechanism (35), although it can be stabilized by posttranscriptional modification. In particular, the C-terminal domain of c-Fos has been shown to be important for protein stability (36). Replacement of serine 362 and serine 374 in the c-Fos C-terminal domain with the phospho-mimetic amino acid aspartic acid protected c-Fos from being degraded, while mutation of these serines to the nonphosphorylatable amino acid alanine decreased c-Fos stability $(11,12,36)$. Importantly, c-Fos protein, which was nearly undetectable in vivo in Rsk2-deficient osteosarcomas as well as in cells isolated from bone tumors of $c$-fos transgenic mice lacking RSK2, was posttranscriptionally regulated by proteasome-mediated degradation. These data suggest that in transformed osteoblasts c-Fos stabilization can be achieved via an RSK2-dependent mechanism. It remains to be determined whether both phosphorylation sites are necessary for c-Fos stabilization as described for fibroblasts $(10,11)$.

The efficiency of c-Fos-dependent osteosarcoma formation was earlier reported to be determined by the levels of c-Fos (24). Moreover, c-Fos has been demonstrated to be necessary for the malignant progression but not for the initiation of skin tumors induced by v-Ha-ras, a strong activator of the ERK pathway (37). We now show that reduced levels of c-Fos affect not the initiation but the progression of osteosarcomas. Increased cell proliferation and reduced cell death were found to be dependent on RSK2 and correlated with c-Fos phosphorylation on serine 362 in osteosarcomas, thereby accounting for the oncogenic activity of c-Fos. The absence of an apparent osteoclast defect in Rsk2deficient mice and the residual phosphorylation on serine 362 in Rsk2-deficient osteoclasts suggest that the decreased osteoclast numbers in c-Fos-induced osteosarcomas are not mediated via c-Fos phosphorylation by RSK2. Moreover, Rsk2 deficiency did not significantly modify the expression of RANKL and its decoy receptor OPG, both in vivo and in in vitro cultures of primary osteoblasts. Therefore, decreased c-Fos levels and the reduced bone formation in the tumor may affect the expression of factors within the tumor, which could account for the observed decrease in osteoclast numbers.

These data demonstrate important roles for RSK2 in normal bone development as well as in c-Fos-dependent osteosarcoma formation. Further analyses of osteoblasts isolated from Rsk2-deficient mice and CLS patients will be required to identify additional RSK2 targets other than ATF4 and c-Fos that are responsible for the observed bone defects. The design of small molecules positively regulating RSK2 activity might allow the generation of therapeutic tools for the treatment of diseases affecting bone remodeling due to decreased osteoblast activity, whereas molecules inhibiting RSK2 activity could be used to treat human osteosarcomas.

\section{Methods}

Mice. All animal studies were approved by the Magistratsabteilung 58, Vienna, Austria. Rsk2 knockout mice (20) were maintained on a C57BL/6J background, and littermates were used for analyses. Homozygous H2-c-fosLTR transgenic mice (32) were crossed with the Rsk2 knockout mice, and most analyses were done on males heterozygous for the fos transgene in the presence or absence of Rsk2.

Cell culture. Primary osteoblasts and osteoclasts were cultured as previously described $(38,39)$. Bone nodule mineralization was analyzed at day 21 of the osteoblast culture by alizarin red staining (Sigma-Aldrich) or staining for alkaline phosphatase activity (ALP kit; Sigma-Aldrich). M-BMMs were generated as previously described (39) in the presence of M-CSF (20 ng/ml) (R\&D Systems Inc.). To induce osteoclast differentiation in coculture, total bone marrow cells $\left(10^{6}\right.$ cells per well $)$ were plated with primary osteoblasts $\left(10^{5}\right.$ cells per well $)$ in 24 -well plates in $\alpha$-modified MEM. The differentiation was induced by addition to the media of dexamethasone $\left(10^{-7} \mathrm{M}\right)$ and dihydroxyvitamin $\mathrm{D}_{3}\left[1 \alpha, 25(\mathrm{OH})_{2} \mathrm{D}_{3}\right]$ $\left(10^{-8} \mathrm{M}\right)$. Immortalized osteosarcoma-derived cell lines and subclones were described elsewhere (6). Primary cells were isolated according to the same procedure from long bone tumors of 10-week-old H2-c-fosLTR/ $R s k 2^{-/ y}$ and $\mathrm{H} 2-c-$ fosLTR/Rsk2+/y littermates.

Western blot and immunoprecipitation. Western blot analyses were performed as previously described (39), and the blots were probed with anti-phospho-RSK2, anti-c-Fos (EMD Biosciences Inc. or Santa Cruz Biotechnology Inc.), or anti- $\beta$-actin (Sigma-Aldrich). The polyclonal anti-phospho-serine 362 c-Fos antibodies were generated against phosphorylated c-Fos C-terminal peptides. For immunoprecipitation, total cell extracts were incubated overnight with a mix of anti-RSK2 antibody (Santa Cruz Biotechnology Inc.) and corresponding agarose-conjugated secondary antibody beads (Sigma-Aldrich).

Immunostaining and immunohistology. For immunostaining, M-BMMs were grown on coverslips, fixed, and stained as previously described (40). Immunoreactivity was revealed using Alexa Fluor-coupled secondary antibodies (Invitrogen Corp.), and DNA was counterstained with DAPI. For immunohistology, 5 - $\mu \mathrm{m}$-thin sections of paraffin-embedded, decalcified bones were incubated overnight at $60^{\circ} \mathrm{C}$, deparaffinized, and boiled twice in antigen-retrieval buffer (DakoCytomation) in a microwave oven (500 watts for 5 minutes). Nonspecific staining was blocked using PBS-containing $2 \% \mathrm{BSA}$ and $10 \%$ normal goat serum.

Proliferation and apoptosis assays. For proliferation curves, triplicate cultures of calvarial osteoblasts were seeded in medium containing $10 \%$ FCS, and cells were counted at the indicated time points. Proliferation indices were determined in vivo or in vitro by immunofluorescence staining using antibodies directed against Ki67 (Novocastra Laboratories Ltd.). Apoptotic indices were determined by TUNEL staining using the In Situ Cell Death Detection Kit (Roche Diagnostics GmbH).

RNase protection assay, Northern blotting, and RT-PCR. Total RNA was isolated using TRIzol reagent (Invitrogen Corp.). c-Fos expression was analyzed using the RNase protection kit $\mathrm{mFos} / \mathrm{Jun}$ protooncogene template set according to the manufacturer's instructions (BD Biosciences - Pharmingen). For Northern blotting, mRNA was purified using the Oligotex Kit (QIAGEN GmbH), and Northern blotting was performed as already described (6). RT-PCR was performed using the following primers: for RANKL, ACCAGCATCAAAATCCCAAG and TTTGAAAGCCCAAAGTACG; for OPG, GGAACCCCAGAGCGAAACACAGT and CTTCTTCCCAGGCAGGCTCTCCAT; for $\beta$-tubulin, CAACGTCAAGACGGCCGTGTG and GACAGAGGCAAACTGAGCACC. 
In situ bybridization. In situ hybridization was performed on sections of decalcified long bones of 2-month-old mice according to standard protocols. Digoxigenin-labeled riboprobes of type I collagen, osteocalcin, osteopontin, and Phex were hybridized overnight at $65^{\circ} \mathrm{C}$. Alkaline phosphatase-conjugated anti-Digoxigenin (Roche Diagnostics $\mathrm{GmbH}$ ) incubation was performed overnight at $4^{\circ} \mathrm{C}$ in a humidified chamber. The signal was visualized using BM Purple AP Substrate (Roche Diagnostics GmbH).

Histology and histomorphometry. After whole-animal contact radiography (Faxitron Cabinet X-Ray System; Faxitron X-Ray Corp.), bones were dissected out and fixed in $3.7 \%$ formaldehyde. After dehydration, undecalcified spines and tibiae were embedded in methyl methacrylate, and $5-\mu \mathrm{m}$ sections were cut in sagittal plane on a rotation microtome. Histomorphometry was performed as previously described (41). For comparative histomorphometry, samples from $R s k 2^{-1 y}$ males, Rsk2-/-females, and sex-matched wild-type mice of the indicated ages were used. Analysis of bone volume (percentage), osteoblast number per bone perimeter (per millimeter), osteocyte number per bone matrix area (per square millimeter), osteoclast number per bone perimeter (per millimeter), cortical thickness (microns), and bone formation rate (cubic micrometers per square micrometer per year) was carried out according to standardized protocols (42) using the OsteoMeasure histomorphometry system (OsteoMetrics Inc.). For the determination of tumor burden, sections of 6 vertebrae of the $\mathrm{H} 2-c-f o s \mathrm{LTR} / R s k 2^{-1 / y}$ group and 8 vertebrae of the H2-c-fosLTR/Rsk $2^{+/ y}$ group were evaluated for tumor area per tissue area (ImageTool; The University of Texas Health Science Center at San Antonio). Statistical analysis was performed using unpaired, 2-sided $t$ test; $P<0.05$ was accepted as significant. Error bars represent the SD. Microcomputed tomography $(\mu \mathrm{CT})$ analysis was used for $3 \mathrm{D}$ histomorphometry and visualization of the vertebral bone structure. Lumbar vertebra L6 of 7-month-old mice was scanned in a $\mu$ CT 40 (SCANCO Medical AG) at a resolution of $6 \mu \mathrm{m}$. Raw data were manually segmented, analyzed with $\mu C T$ Evaluation Program version 4.4A, and displayed in $\mu$ CT Ray version

1. Karsenty, G., and Wagner, E.F. 2002. Reaching a genetic and molecular understanding of skeletal development. Dev. Cell. 2:389-406.

2. Jochum, W., Passegue, E., and Wagner, E.F. 2001. $\mathrm{AP}-1$ in mouse development and tumorigenesis. Oncogene. 20:2401-2412.

3. Eferl, R., and Wagner, E.F. 2003. AP-1: a doubleedged sword in tumorigenesis. Nat. Rev. Cancer. 3:859-868.

4. Wang, Z.Q., et al. 1992. Bone and haematopoietic defects in mice lacking c-fos. Nature. 360:741-745.

5. Johnson, R.S., Spiegelman, B.M., and Papaioannou, V. 1992. Pleiotropic effects of a null mutation in the c-fos proto-oncogene. Cell. 71:577-586.

6. Grigoriadis, A.E., Schellander, K., Wang, Z.Q., and Wagner, E.F. 1993. Osteoblasts are target cells for transformation in c-fos transgenic mice. J. Cell Biol. 122:685-701.

7. Pearman, A.T., Chou, W.Y., Bergman, K.D., Pulumati, M.R., and Partridge, N.C. 1996. Parathyroid hormone induces $\mathrm{c}$-fos promoter activity in osteoblastic cells through phosphorylated cAMP response element (CRE)-binding protein binding to the major CRE. J. Biol. Chem. 271:25715-25721.

8. McCauley, L.K., Koh, A.J., Beecher, C.A., and Rosol, T.J. 1997. Proto-oncogene c-fos is transcriptionally regulated by parathyroid hormone (PTH) and PTH-related protein in a cyclic adenosine monophosphate-dependent manner in osteoblastic cells. Endocrinology. 138:5427-5433.

9. Deng, T., and Karin, M. 1994. c-Fos transcriptional activity stimulated by $\mathrm{H}$-Ras-activated protein kinase distinct from JNK and ERK. Nature. 371:171-175.

10. Chen, R.H., Juo, P.C., Curran, T., and Blenis, J. 1996. Phosphorylation of c-Fos at the C-terminus enhances its transforming activity. Oncogene.
3.0 (SCANCO Medical AG). To assess the femoral thickness, 15 planes per femur were scanned at the midshaft. Longitudinal femoral thickness was measured with the Distance3D tool of $\mu \mathrm{CT}$ Evaluation Program version 4.4A. Tumor incidence was evaluated by scanning of 20 tail vertebrae per group. For biomechanical testing, femurs were dissected and a 3-point bending was performed as previously described (41).

\section{Acknowledgments}

We are very grateful to Andre Hanauer for the Rsk2 knockout mice, Marc Piechaczyk for the c-Fos mutant cDNAs, and Claudine Oudet for the human Phex cDNA. We also thank very much Karl Mechtler and Harald Scheuch for help in generating the anti-c-Fos phospho-serine 362 antibody, Uta Möhle-Steinlein and Robert Eferl for technical advice, Thomas Decker, Koichi Matsuo, and Astrid Höbertz for critical reading of the manuscript, and Agi Grigoriadis and Maria Sibilia for numerous helpful suggestions. Latifa Bakiri is the recipient of a European Molecular Biology Organization long-term fellowship and Arndt Schilling of a fellowship of the Erich and Gertrud Roggenbuck Foundation. The Research Institute of Molecular Pathology is supported by Boehringer Ingelheim.

Received for publication July 30, 2004, and accepted in revised form January 4, 2005.

Address correspondence to: Erwin F. Wagner, Research Institute of Molecular Pathology, Dr. Bohr Gasse 7, A-1030 Vienna, Austria. Phone: 43-1-797-30-888; Fax: 43-1-798-9390; E-mail: Wagner@imp.univie.ac.at.

Jean-Pierre David and Denis Mehic contributed equally to this work.
12:1493-1502.

11. Okazaki, K., and Sagata, N. 1995. The Mos/MAP kinase pathway stabilizes c-Fos by phosphorylation and augments its transforming activity in NIH $3 \mathrm{~T} 3$ cells. EMBO J. 14:5048-5059.

12. Murphy, L.O., Smith, S., Chen, R.H., Fingar, D.C., and Blenis, J. 2002. Molecular interpretation of ERK signal duration by immediate early gene products. Nat. Cell Biol. 4:556-564.

13. Takayanagi, H., et al. 2002. RANKL maintains bone homeostasis through c-Fos-dependent induction of interferon-beta. Nature. 416:744-749.

14. Nebreda, A.R., and Gavin, A.C. 1999. Perspectives: signal transduction. Cell survival demands some Rsk. Science. 286:1309-1310.

15. De Cesare, D., Jacquot, S., Hanauer, A., and Sassone-Corsi, P. 1998. Rsk-2 activity is necessary for epidermal growth factor-induced phosphorylation of CREB protein and transcription of c-fos gene. Proc. Natl. Acad. Sci. U. S. A. 95:12202-12207.

16. Bruning, J.C., et al. 2000. Ribosomal subunit kinase- 2 is required for growth factor-stimulated transcription of the c-Fos gene. Proc. Natl. Acad. Sci. U. S. A. 97:2462-2467.

17. Cheung, P., et al. 2000. Synergistic coupling of histone $\mathrm{H} 3$ phosphorylation and acetylation in response to epidermal growth factor stimulation. Mol. Cell. 5:905-915.

18. Hanauer, A., and Young, I.D. 2002. Coffin-Lowry syndrome: clinical and molecular features. J. Med. Genet. 39:705-713.

19. Dufresne, S.D., et al. 2001. Altered extracellular signal-regulated kinase signaling and glycogen metabolism in skeletal muscle from p90 ribosomal S6 kinase 2 knockout mice. Mol. Cell. Biol. 21:81-87.

20. Yang, X., et al. 2004. ATF4 is a substrate of RSK2 and an essential regulator of osteoblast biol- ogy: implication for Coffin-Lowry Syndrome. Cell. 117:387-398.

21. Anonymous. 1995. A gene (PEX) with homologies to endopeptidases is mutated in patients with $\mathrm{X}$-linked hypophosphatemic rickets. The HYP Consortium. Nat. Genet. 11:130-136.

22. Econs, M.J., et al. 1998. A PHEX gene mutation is responsible for adult-onset vitamin D-resistant hypophosphatemic osteomalacia: evidence that the disorder is not a distinct entity from X-linked hypophosphatemic rickets. J. Clin. Endocrinol. Metab. 83:3459-3462.

23. Strom, T.M., et al. 1997. Pex gene deletions in Gy and Hyp mice provide mouse models for X-linked hypophosphatemia. Hum. Mol. Genet. 6:165-171.

24. Wang, Z.Q., Liang, J., Schellander, K., Wagner, E.F., and Grigoriadis, A.E. 1995. c-fos-induced osteosarcoma formation in transgenic mice: cooperativity with c-jun and the role of endogenous c-fos. Cancer Res. 55:6244-6251.

25. Vahle, J.L., et al. 2002. Skeletal changes in rats given daily subcutaneous injections of recombinant human parathyroid hormone (1-34) for 2 years and relevance to human safety. Toxicol. Pathol. 30:312-321.

26. Alessi, D.R. 1997. The protein kinase C inhibitors Ro 318220 and GF $109203 \mathrm{X}$ are equally potent inhibitors of MAPKAP kinase-1beta (Rsk-2) and p70 S6 kinase. FEBS Lett. 402:121-123.

27. Fleischmann, A., et al. 2003. Impaired long-term memory and NR2A-type NMDA receptor-dependent synaptic plasticity in mice lacking c-Fos in the CNS. J. Neurosci. 23:9116-9122.

28. Quarles, L.D. 2003. FGF23, PHEX, and MEPE regulation of phosphate homeostasis and skeletal mineralization. Am. J. Physiol. Endocrinol. Metab. 285:E1-E9. 
29. Miao, D., et al. 2001. Osteomalacia in hyp mice is associated with abnormal phex expression and with altered bone matrix protein expression and deposition. Endocrinology. 142:926-939.

30. Meyer, R.A., Jr., Young, C.G., Meyer, M.H., Garges, P.L., and Price, D.K. 2000. Effect of age on the expression of Pex (Phex) in the mouse. Calcif. Tissue Int. 66:282-287.

31. Ruchon, A.F., et al. 2000. Developmental expression and tissue distribution of Phex protein: effect of the Hyp mutation and relationship to bone markers. J. Bone Miner. Res. 15:1440-1450.

32. Ruther, U., Komitowski, D., Schubert, F.R., and Wagner, E.F. 1989. c-fos expression induces bone tumors in transgenic mice. Oncogene. 4:861-865.

33. Papachristou, D.J., Batistatou, A., Sykiotis, G.P., Varakis, I., and Papavassiliou, A.G. 2003. Activation of the JNK-AP-1 signal transduction pathway is associated with pathogenesis and progression of human osteosarcomas. Bone. 32:364-371.

34. Grigoriadis, A.E., et al. 1994. c-Fos: a key regulator of osteoclast-macrophage lineage determination and bone remodeling. Science. 266:443-448.

35. Acquaviva, C., et al. 2001. Identification of a C-terminal tripeptide motif involved in the control of rapid proteasomal degradation of c-Fos protooncoprotein during the $\mathrm{G}(0)$-to-S phase transition. Oncogene. 20:7563-7572.

36. Ferrara, P., et al. 2003. The structural determinants responsible for c-Fos protein proteasomal degradation differ according to the conditions of expression. Oncogene. 22:1461-1474.

37. Saez, E., et al. 1995. c-fos is required for malignant progression of skin tumors. Cell. 82:721-732.

38. Jochum, W., et al. 2000. Increased bone formation and osteosclerosis in mice overexpressing the transcription factor Fra-1. Nat. Med. 6:980-984

39. David, J.P., Sabapathy, K., Hoffmann, O., Idar- raga, M.H., and Wagner, E.F. 2002. JNK1 modulates osteoclastogenesis through both c-Jun phosphorylation-dependent and -independent mechanisms. J. Cell Sci. 115:4317-4325.

40. Bakiri, L., Lallemand, D., Bossy-Wetzel, E., and Yaniv, M. 2000. Cell cycle-dependent variations in c-Jun and JunB phosphorylation: a role in the control of cyclin D1 expression. EMBO J. 19:2056-2068.

41. Amling, M., et al. 1999. Rescue of the skeletal phenotype of vitamin D receptor-ablated mice in the setting of normal mineral ion homeostasis: formal histomorphometric and biomechanical analyses. Endocrinology. 140:4982-4987.

42. Parfitt, A.M., et al. 1987. Bone histomorphometry: standardization of nomenclature, symbols, and units. Report of the ASBMR Histomorphometry Nomenclature Committee. J. Bone Miner. Res. 2:595-610. 